\title{
Supranumerários bilaterais em mandíbula: relato de caso
}

\author{
Bilateral supernumerary mandibular:case report \\ Dientes supernumerarios mandibulares:reporte de un caso \\ Vítor Bruno TESLENCO ${ }^{1}$ \\ Ellen Cristina GAETTI JARDIM ${ }^{2}$ \\ Júlio César Leite da SILVA ${ }^{2}$
}

${ }^{1}$ Cirurgião-Dentista pela Faculdade de Odontologia, UFMS, 79070-900, Campo Grande-MS, Brasil

${ }^{2}$ Professor(a) Adjunto da Disciplina de Cirurgia e Traumatologia Buco-Maxilo-Facial,

Faculdade de Odontologia, UFMS, 79070-900, Campo Grande-MS, Brasil

\begin{abstract}
Resumo
O aparecimento de dentes supranumerários na arcada dentária é denominado hiperdontia. Tal patologia ainda não apresenta etiologia definida e seu diagnóstico é feito, geralmente, por exames de rotina. Sua prevalência é maior em região de maxila (pré-maxila), sendo os homens mais afetados em uma proporção de 2:1. O propósito do presente trabalho foi relatar caso clínico com tratamento da patologia por meio de exérese do elemento supranumerário, a fim de manter a integridade das estruturas adjacentes ao elemento. Paciente de 23 anos, do sexo feminino, procurou às clínicas da Faculdade de Odontologia (FAODO) da Universidade Federal de Mato Grosso do Sul para avaliação e conduta de sua patologia. A cirurgia dos supranumerários foi realizada em duas etapas, sendo a primeira no dia 07/12/2015 e a segunda 01/02/2016. Concluiu-se que se faz necessária a realização de exames radiográficos de rotina para se controlar a presença destas anomalias. Se bem planejado e indicado pelo profissional, o tratamento cirúrgico se mostra eficiente para a resolução dos casos.
\end{abstract}

Descritores: Dente Impactado; Dente Supranumerário; Cirurgia Bucal.

\begin{abstract}
The appearance of supernumerary teeth in the dental arch is called hyperdontia. This pathology has not defined etiology and diagnosis is done usually by routine tests. Its prevalence is higher in the jaw region (pre-jaw), being the most affected men at a ratio of 2: 1 . The purpose of this paper was related the treatment of disease by excision of the supernumerary element in order to maintain the integrity of structures adjacent to the element. A 23 year old female patient sought to clinics Dental School (FAODO) of the Federal University of Mato Grosso do Sul for evaluation and management of their disease. Surgery of the supernumerary was performed in two stages, the first on 12/07/2015 and the second 02/01/2016. It's concluded that the performance of routine radiographs is necessary to control the presence of these anomalies. If well planned and directed by professional the treatment by surgical removal is efficient for the resolution of cases.
\end{abstract}

Descriptors: Tooth, Impacted; Tooth, Supernumerary; Surgery, Oral.

\section{Resumen}

Lo surgimiento de dientes supernumerarios en el arco dental se llama hiperdoncia. Esta patología no ha definida la etiología. El diagnóstico se realiza generalmente mediante pruebas de rutina. Su prevalencia es mayor en la región de la maxila, siendo los hombres más afectados en una proporción de 2: 1. El objetivo del presente trabajo es reportar el tratamiento de la enfermedad por escisión del elemento supernumerario con el fin de mantener la integridad de las estructuras adyacentes. Una mujer con 23 años de edad llegaron a las clínicas de la Facultad de Odontología (FAODO) de la Universidad Federal de Mato Grosso do Sul, para la evaluación y control de la enfermedad. La cirugía del supernumerario se realizó en dos etapas, la primera en 12/07/2015 y la segunda en 01/02/2016. Las radiografías de rutina son necesarias controlar la presencia de estas anomalías. Si bien planificada y dirigida por el profesional, el tratamiento quirúrgico es pantallas eficaces para la resolución de los casos.

Descriptores: Diente Impactado; Diente Supernumerario; Cirugía Bucal.

\section{INTRODUÇÃO}

Inúmeras são as anomalias que acometem a cavidade bucal, sendo a de número por presença de elementos supranumerários, denominada hiperdontia. São classificados de acordo com sua localização anatômica, sendo os mesiodens, aqueles localizados na região de incisivos centrais superiores, temos os paramolares ou distomolares quando sua localização se dá lateralmente ou distalmente ao $3^{\circ}$ molar, respectivamente. São mais comumente encontrados unilateralmente, porém quando ocorrem de maneira bilateral, sua prevalência é maior em região de prémolares mandibulares ${ }^{1,2}$.

Sua etiologia ainda não foi bem elucidada, porém, existem diversas teorias referentes a ela, como a teoria da hiperatividade da lâmina dentária na fase de iniciação, onde seria gerado um terceiro germe dentário; a teoria atávica, que seria o reaparecimento de uma característica ancestral.
Pacientes portadores de Displasia Cleidocraniana e Síndrome de Gardner apresentam comumente hiperdontia ${ }^{3-7}$. Quanto a sua morfologia podem ser classificados em suplementares e rudimentares. São denominados suplementares quando suas características se assemelham à dentição normal. Já os rudimentares, são aqueles que apresentam discrepâncias morfológicas significativas, podendo ser menores ou ainda conóides ${ }^{8,9}$.

O diagnóstico precoce é essencial para realização de um tratamento adequado e, consequentemente, prevenção de algumas possíveis complicações, tais como reabsorção radicular dos dentes adjacentes, impacção, apinhamento dental, diastemas, lesões císticas, retardos na erupção, necrose pulpar e abscesso dento-alveolar. Geralmente o diagnóstico é feito por exames de rotina como a ortopantomografia, visto que em grande parte dos casos a 
presença do supranumerário é assintomática ${ }^{10,11}$

O termo "impactação" pode ser utilizado para todo e qualquer dente que não tenha irrompido na cavidade oral no tempo esperado.

Esse impedimento da erupção pode-se dar em razão de dentes adjacentes, excesso de tecido mole, recobrimento por osso denso ou ainda por uma anormalidade genética que evita a erupção. Os dentes impactados mais comuns são os terceiros molares maxilares e mandibulares, seguidos pelos caninos maxilares e pré-molares mandibulares. Em casos de impactação, cerca de $75 \%$ dos dentes irão irromper espontaneamente após a remoção do supranumerário, dependendo de quatro fatores, sendo esses: tipo de dente supranumerário, o grau de deslocamento e inclinação do dente não irrompido, o espaço avaliado para erupção, tempo de diagnóstico e de intervenção cirúrgica ${ }^{12,13}$.

O tratamento pode variar, de acordo com a posição e localização do dente e as estruturas adjacentes a ele. O momento certo para sua remoção é após a formação completa da raiz do dente adjacente ao supranumerário. A remoção do dente supranumerário estará indicada em algumas ocasiões como em casos de inibição ou atraso na erupção dos incisivos centrais, sua erupção espontânea já tenha acontecido, quando há associação a patologias. Porém, a indicação de exérese ou não dos dentes extranumerários vai depender do custo-benefício avaliado pelo profissional $^{11-14}$.

A prevalência é maior nos homens em uma relação de 2:1. O aparecimento destes dentes é mais frequente na maxila, principalmente a região anterior, em uma relação maxilo-mandíbula numa proporção de $10: 1^{1,3}$. No presente relato, a presença desta anomalia acometeu uma paciente do sexo feminino, em região de pré-molares mandibulares.

\section{CASO CLÍNICO}

A paciente do sexo feminino com 23 anos de idade compareceu às clinicas da Faculdade de Odontologia (FAODO) da Universidade Federal do Mato Grosso do Sul para realizar tratamento odontológico. A mesma apresentou em seu exame ortopantomográfico de rotina a presença de dentes supranumerários em região de pré-molares mandibulares, bilateralmente. A mesma não apresentava sintomatologia, queixas álgicas com relação a presença do supranumerário e estava classificada como sendo uma paciente do tipo ASA I, não apresentando histórico de reações alérgicas aos medicamentos e materiais que seriam utilizados para o procedimento. Ao exame clínico observouse discreto abaulamento na região lingual dos dentes 45,46 , 35 e 36 (Figura 1).

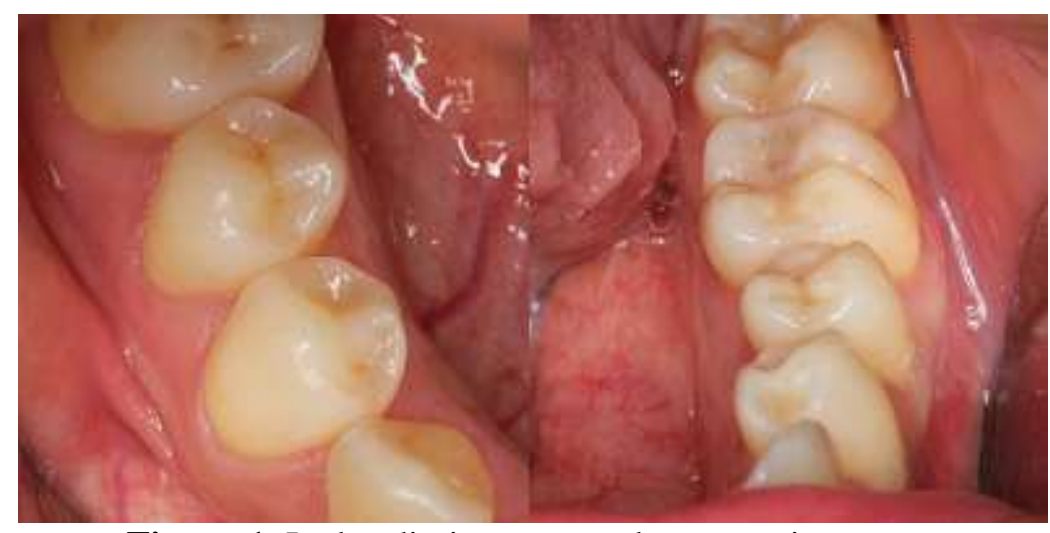

Figura 1: Lados direito e esquerdo, respectivamente, evidenciando o discreto abaulamento na região lingual, entre segundos pré-molares e primeiros molares, bilateralmente.

Ao exame tomográfico, através de cortes coronais comprovou-se a localização de ambos os dentes pela superfície lingual, sendo que no lado esquerdo, havia contato entre o dente supranumerário e o elemento dentário 35. Devido à presente impactação do elemento extranumerário, optou-se pelo tratamento através de exodontia do mesmo (Figura 2).

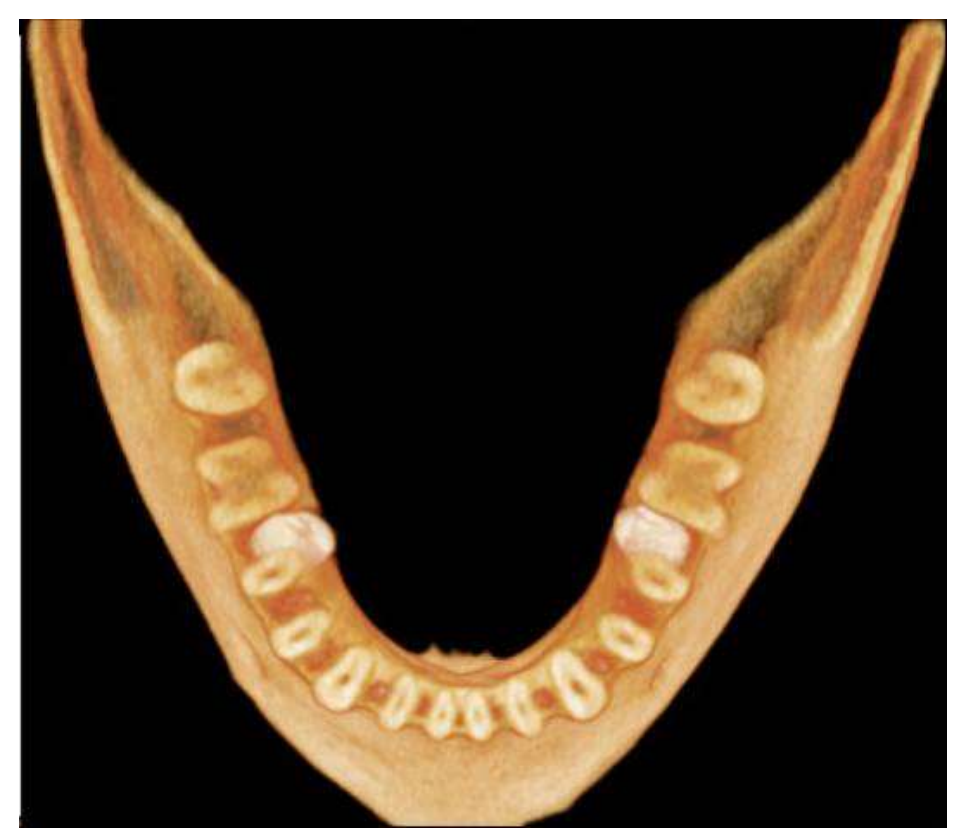

Figura 2: Corte axial de reconstrução 3D, em vista oclusal denotando a presença dos dentes supranumerários lingualmente, entre segundos pré-molares e primeiros molares

A medicação pré-operatória utilizada foi a dexametasona 4mg (EMS Pharma (C) - dois comprimidos uma hora antes do procedimento e a utilização de digluconato de clorexidina 0,12\% (Periogard - ColgatePalmolive Company (C) na forma de bochecho, durante os três dias que precederam o procedimento.

A primeira cirurgia (lado direito) foi realizada no dia 07/12/2015 e a segunda (lado esquerdo) no dia 01/02/2016, sendo ambas na clínica da disciplina de Cirurgia e Traumatologia Buco-maxilo-facial da Faculdade de Odontologia (FAODO) da Universidade Federal do Mato Grosso do Sul. O primeiro procedimento decorreu das 8 às 9:20 horas sem intercorrências, tendo como início a antissepsia intra-oral com digluconato de clorexidina a $0,12 \%$ e extra-oral com digluconato de clorexidina a $2 \%$ (Maquira Indústria de Produtos Odontológicos 1tda (C). Foi realizada anestesia tópica com Benzocaína 20\% (DFL Indústria e Comércio S.A. (C), aplicada em mucosa seca, por 2 minutos. Em seguida foi executada anestesia por bloqueio regional do nervo Mentoniano direito e incisivo, e do tipo terminal infiltrativa subperiostal em região de soalho bucal direito com Articaína 4\% (DFL Indústria e Comércio S.A. (C) com epinefrina 1:100.000, sendo que a agulha utilizada foi a curta de $23 \mathrm{~mm}$ de comprimento e calibre de $30 \mathrm{G}$ (Septodont do Brasil Ltda ( )).

Realizou-se uma incisão intrasulcular por lingual e retalho do tipo envelope com cabo de bisturi número 3 (Golgran ${ }^{\circledR}$ ) e lâmina 15C (Lamedid (C)). O retalho estendeuse da região lingual do incisivo central inferior até primeiro molar ipsilateral. Foi realizado descolamento mucoperiostal com auxílio de descolador de Molt simples (Golgran (®), seguido de ostectomia com broca 702 HL (JET Carbide Burs, Labordental Ltda. (C) em caneta de alta rotação (Dabi Atlante (C) simultaneamente com irrigação assistida de soro fisiológico 0,9\%. (Figura 3).

Para que pudesse ser mantido o espaço biológico e para evitar recessões gengivais, no momento da ostectomia, foram tomadas as devidas precauções de manutenção de pelo menos $3 \mathrm{~mm}$ de osso remanescente entre a crista óssea alveolar e o bordo superior da loja cirúrgica.

Para facilitar a remoção do elemento dentário e para menor morbidade à paciente, optou-se pela odontossecção 
que foi realizada no sentido perpendicular ao longo eixo do dente, separando a coroa e raiz. Removeu-se o fragmento radicular com alavanca Seldin Reta (Golgran () ) e o capuz peri-coronário com cureta de Lucas número 85 e pinça hemostática curva (Golgran $\left.{ }^{\circledR}\right)$. Realizou-se irrigação abundante com soro fisiológico $0,9 \%$, a fim de se remover qualquer tipo de resíduos, regularização dos bordos da loja cirúrgica com lima para osso Seldin 11 (Golgran $($ ) ) e reposicionamento/sutura do retalho com fio de Nylon 5-0 com agulha circular $3 / 8$ de 2,0 cm (Procare $\left.{ }^{\circledR}\right)$. (Figura 4).

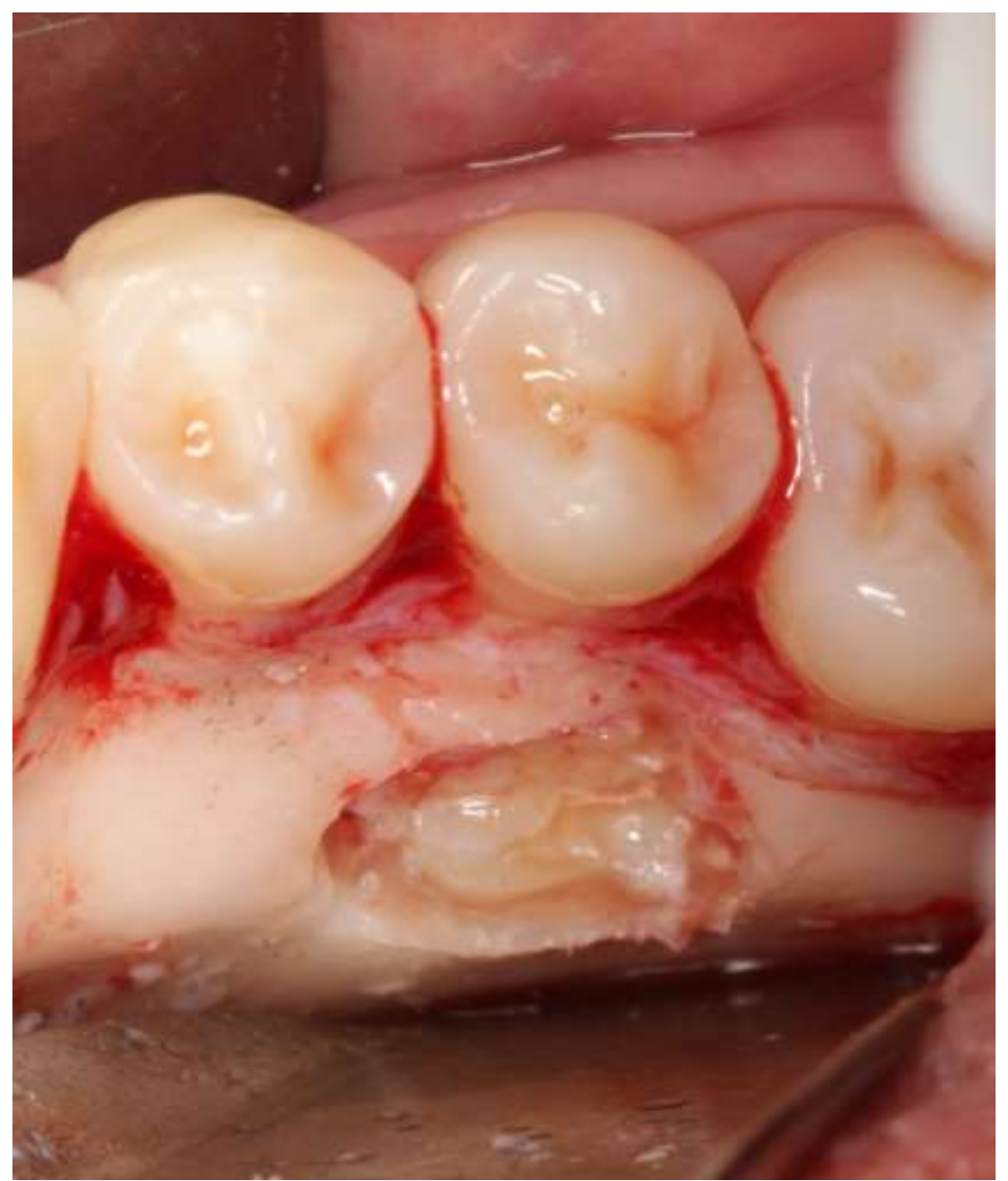

Figura 3: Retalho do tipo envelope com exposição do supranumerário do lado direito.

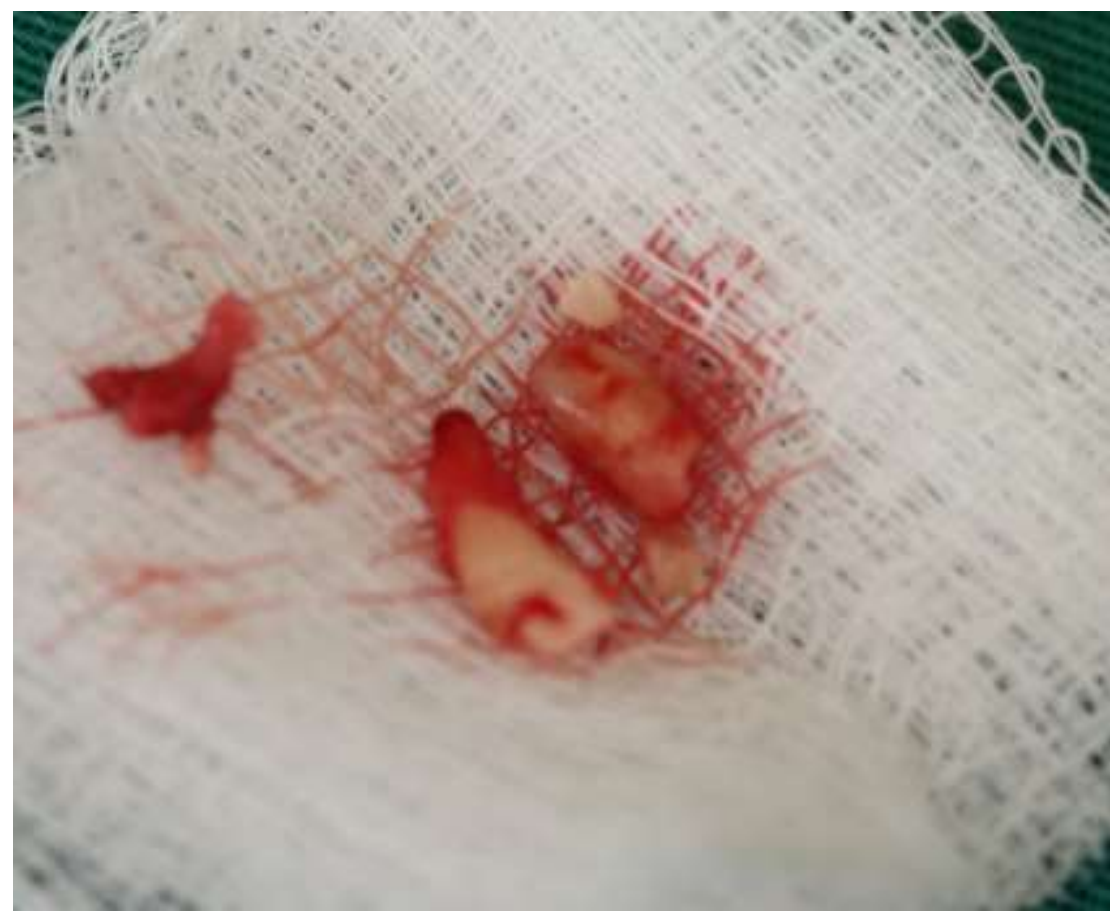

Figura 4: Dente removido do lado direito.

O segundo procedimento decorreu das 7:30horas às 8:20horas, também sem intercorrências, de maneira semelhante ao primeiro. Procedimento cirúrgico comumente realizado em clínicas odontológicas (Figuras 5 e 6 ).

Em ambas as cirurgias foram administrados no pósoperatório cetorolaco de trometamol 10mg (Toragesic ${ }^{\circledR}$ ) via sublingual de 8 em 8 horas, por 3 dias, como medicamento analgésico e antiinflamatório.

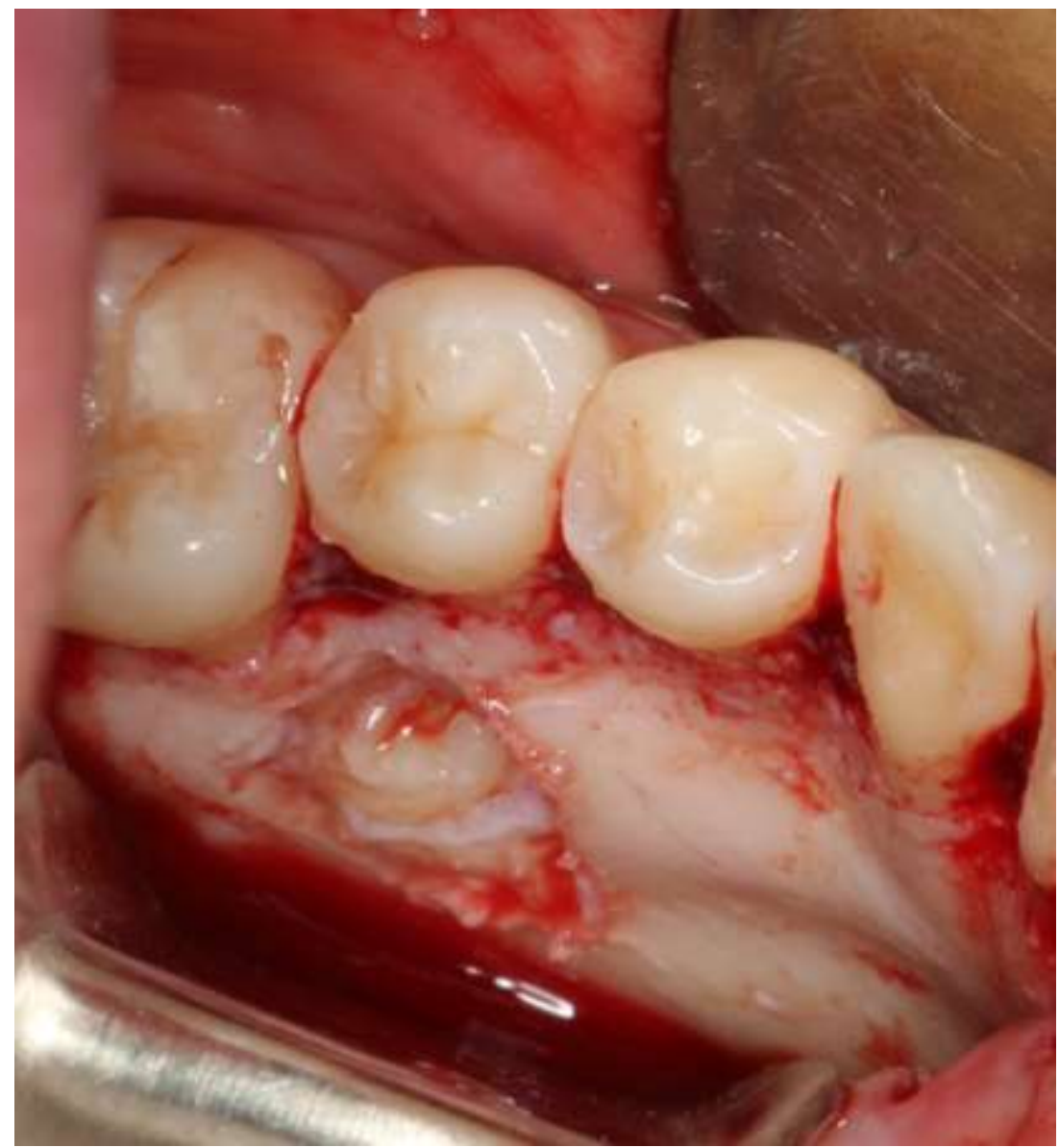

Figura 5: Retalho do tipo envelope com exposição do supranumerário do lado esquerdo.

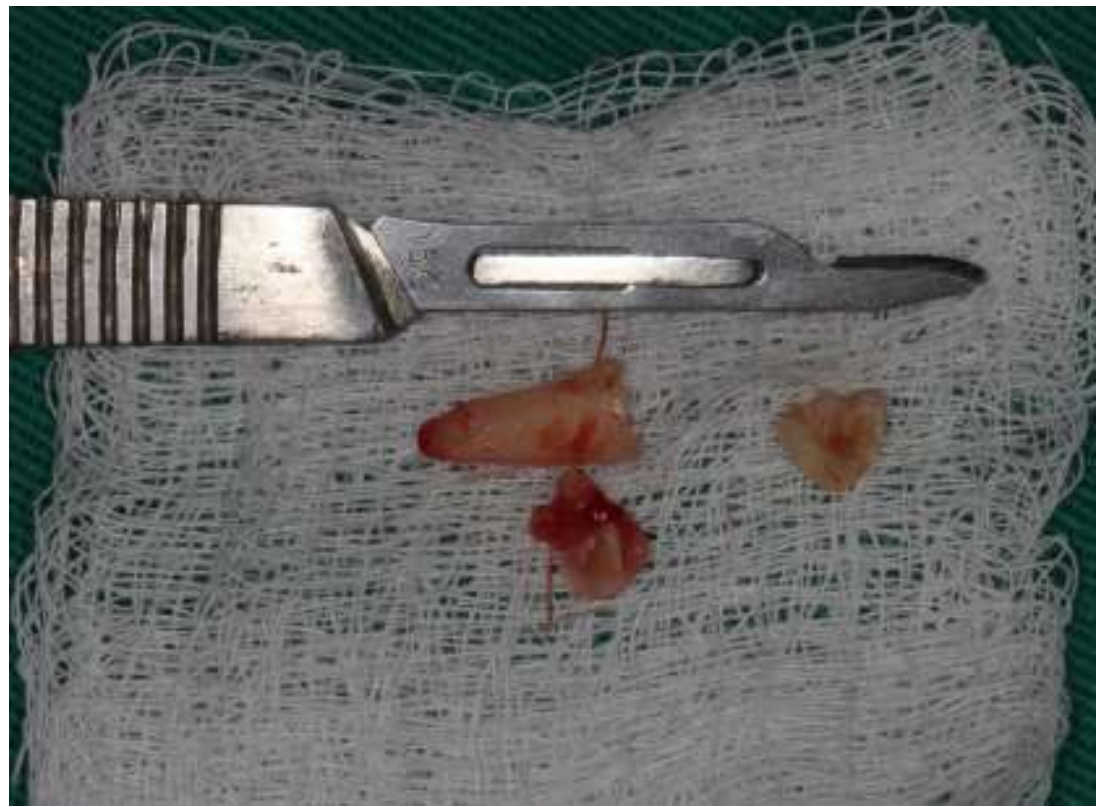

Figura 6: Dente removido do lado esquerdo.

\section{DISCUSSÃO}

O diagnóstico precoce da presença de dentes supranumerários é essencial. No presente trabalho o local onde os elementos dentários foram encontrados foi na mandíbula, mais precisamente região entre segundos prémolares e primeiros molares bilateralmente, o que difere do encontrado na literatura de que a proporção destes dentes encontrados na maxila é maior em uma relação de 10:1 com relação à mandíbula. Porém, sua presença ocorre bilateralmente mais comumente na região anatômica encontrada no presente trabalho. Com relação ao sexo o encontrado neste relato não condiz com a proporção do masculino de 2:1 encontrada por Neville et al. ${ }^{1}$. Assim como a presença dos elementos se deu na dentição permanente ${ }^{1,3}$.

O momento ideal para remoção destes elementos será quando a formação das raízes dos dentes adjacentes estiver completa, o que diminui a chance de causar danos a essas estruturas. A paciente já apresentava os dentes supranumerários em completa formação, assim como os elementos dentários adjacentes a ele $\mathrm{e}^{15,16,18}$.

A prevalência do aparecimento destes elementos supranumerários pode variar entre $0,5 \%$ e $3,8 \%$. Por isso, a 
ortopantomografia é um instrumento essencial para o achado de tais elementos e deve ser um exame de rotina na prática odontológica. Ela deve ser feita de maneira periódica a partir dos seis anos de idade, a fim de se evitar o diagnóstico tardio dessas anomalias e preservar o desenvolvimento normal de todo o sistema estomatognático ${ }^{15,17-23}$.

A escolha do exame tomográfico foi realizada para que se pudesse melhorar a precisão cirúrgica, com relação à localização, posicionamento e acesso cirúrgico do elemento supranumerário. Faz-se necessário lançar mão de recursos complementares como a tomografia para que o cirurgião possa planejar e prever com maior eficiência a qualidade dos resultados do procedimento proposto.

\section{CONCLUSÃO}

$\mathrm{O}$ tratamento proposto à paciente se mostrou eficaz para a resolução do caso, uma vez que a mesma teve um pós-operatório satisfatório em cada um dos procedimentos. Devido a manutenção da cadeia asséptica através da utilização de campos estéreis e bochecho com digluconato de clorexidina $0,12 \%$, não foi necessário a utilização de antibioticoterapia e a paciente não apresentou quadro de infecção pós-operatória. Foi possível então, através da remoção dos elementos, evitar problemas futuros à paciente, como o surgimento de patologias, reabsorções radiculares, necrose dos dentes adjacentes.

\section{REFERÊNCIAS}

1. Neville BW, Damm DD. Patologia oral e maxilofacial. $2^{a}$ ed. Rio de Janeiro: Guanabara Koogan; 2004.

2. Almeida TE, Saavedra Jr J, Kawakami PY, Palis CA, Mariani PB, Dottore AM. Hiperdontia: Relato de Caso com 8 Elementos Supranumerários. Rev Odontol Univ Cid São Paulo. 2010; 22(1): 78-84.

3. Regezi JA, Sciubba JS, Jordan RCK. Patologia bucal: correlações clinicopatológicas. 6 ed. Rio de Janeiro: Elsevier; 2012.

4. Prismosch RE. Anterior supernumerary teethassessmentand surgical intervention in children. Pediatr Dent. 1981; 3(2):204-15.

5. Santos AP, Ammari MM, Moliterno LF, Júnior JC. First report of bilateral supernumerary teeth associated with both primary and permanent maxillary canines. J Oral Sci. 2009; 51(1):145-50.

6. Almeida RR, Isbralde CMB, Ramos AL, Terada $\mathrm{HH}$, Ribeiro R, Carreiro LS. Supranumerários - Implicações e procedimentos clínicos. Rev Dental Press Ortodon Ortop Maxilar. 1997; 2(6):91-108.

7. Reis LFG, Giovanini A, Namba EL, Silva ELFM, Garcia MA. Dentes supranumerários retidos interferindo no tratament ortodôntico. RSBO. 2006; 3(2):20-5.

8. Azenha MR, Zorzetto DLG, Marzola C, Moura LA, Handem RH. Abordagem Cirúrgica de Dente Supranumerário (Mesiodens) na Região Palatina: Caso Clínico. Rev Port Estomatol Cir Maxilofac, 2007;48(1):37-41.

9. Lobato AMR, Columbano JN, Souza MMG. Hiperdontia na região de incisivos superiores. J Bras Ortodon Ortop Facial, Curitiba. 2002; 7(41):389-96.

10. Moura WL, Cravinhos JCP, Moura CDVS, Freire SASR, Monteiro AMO, Pinheiro SDA, Rodrigues WFB. Prevalência de dentes supranumerários em pacientes atendidos no Hospital Universitário da UFPI: um estudo retrospectivo de cinco anos. Rev Odontol UNESP. 2013; 42(3): 167-71.
11. Fernandes AV, Rocha NS, Almeida RAC, e Silva EDO, Vasconcelos BCE. Quarto molar incluso: relato de caso. Rev Cir Traumatol Buco-Maxilo-Fac. 2005; 5(2):61-6.

12. Hupp JR, Ellis $3^{\circ}$ E, Tucker MR. Cirurgia oral e maxillofacial contemporânea. $5^{\mathrm{a}}$ ed. Rio de Janeiro: Elsevier; 2009.

13. Hattab FN, Yassin OM, Rawashdeh MA. Supernumerary teeth: Report of three cases and review of literature. ASDC J Dent Child. 1994; 61(5-6):382-93.

14. Cochrane SM, Clark JR, Hunt NP. Late Developing Supernumerary Teeth in the Mandible. Br J Orthod. 1997; 24(4):293-6.

15. Dayube AC, Pompermayer L, Pena NNS. Levantamento das anomalias dentárias de número (supranumerários) em radiografias panorâmicas de um serviço de documentação odontológica da cidade do SalvadorBahia. R Ci méd biol. 2011; 10(1):34-8.

16. Cunha Filho JJ, Puricelli E, Hennigen TW, Leite MGT, Pereira MA, Martins GL. Ocorrência de dentes supranumerários em pacientes do serviço de Cirurgia e Traumatologia Buco-Maxilo-Facial, Faculdade de Odontologia da UFRGS, no período de 1998 a 2001. R Fac Odontol, Porto Alegre. 2002; 43(2):27-34.

17. Carvalho S, Mesquita P, Afonso A. Prevalência das anomalias de número numa população portuguesa. Estudo radiográfico. Rev Port Estomatol Med Dental Cir Maxilofac. 2011; 52(1):7-12.

18. Soares AB, Ferreira Filho UG, Marzola C, Toledo Filho JL, Barbosa JL, Haagsma IB. Prevalência de Dentes Supranumerários no Município de Curitiba, Paraná, Brasil e suas Posições no Arco Dental pelo Exame de 3000 Ortopantomografias. Rev. Bras Cir Buco-MaxiloFac. 2010; 10(1):23-32.

19. Leite Segundo AV, de Faria DLB, da Silva UH, Vieira ITA. Estudo epidemiológico de dentes supranumerários diagnosticados pela radiografia panorâmica. Rev Cir Traumatol. Buco-Maxilo-Fac. 2006;6(2):53-6.

20. Corrêa FG, Ferreira FV, Friedrich LR, Pistóia AD, Pistóia GD. Prevalência de dentes supranumerários estudo retrospectivo. Int J Dent. 2009; 8(1):11-5.

21. Nunes KM, Medeiros MV, Ceretta LB, Simões PW, Azambuja FG, Sônego FGF, Pires PDS. Dente supranumerário: revisão bibliográfica e relato de caso clínico. Rev Odontol Univ Cid São Paulo, 2015; 27(1):72-8.

22. Negrete D, Carvalho PEG, Fuziy A, Torres FC, Triviño T, Flaiban E. Prevalência de dentes supranumerários em pacientes de Ortodontia. Rev Odontol Univ Cid São Paulo. 2015; 27(1):6-13.

23. Bezerra PKM, Bezerra PM, Cavalcanti AL. Dentes supranumerários: revisão de literatura e relato de caso. $\mathrm{R}$ Ci méd biol. 2007; 6(3): 349-56. 


\section{CONFLITO DE INTERESSES}

Os autores declaram não haver conflitos de interesse.

\section{AUTOR PARA CORRESPONDÊNCIA}

Ellen Cristina Gaetti Jardim

ellengaetti@gmail.com

Submetido em 13/12/2016

Aceito em 01/01/2017 